\title{
Percutaneous Cryoablation of Renal Tumors: Initial Indian Experience
}

\author{
Ujjwal Gorsi ${ }^{1}$ Rishabh Jain ${ }^{1}$ Akash Bansal ${ }^{1}$ Naveen Kalra ${ }^{1}$ Mandeep Kang ${ }^{1}$ \\ Sreedhara B. Chaluvashetty ${ }^{1}$ Ravimohan Suryanarayan Mavuduru ${ }^{2}$ Santosh Kumar ${ }^{2}$ \\ Shrawan K. Singh ${ }^{2}$ Manavjit Singh Sandhu ${ }^{1}$
}

${ }^{1}$ Department of Radiodiagnosis, Postgraduate Institute of Medical

Education and Research (PGIMER), Chandigarh, India

2 Department of Urology, Postgraduate Institute of Medical Education

and Research (PGIMER), Chandigarh, India

Address for correspondence Ujjwal Gorsi, MD, Department of

Radiodiagnosis, PGIMER, Sector 12, Chandigarh, India

(e-mail: ujjwalgorsi@gmail.com).

J Clin Interv Radiol ISVIR 2022;6:163-169.

\begin{abstract}
Keywords

- cryoablation

- renal cell carcinoma

- ablation

Purpose The purpose of this study was to report short-term outcomes of cryoablation of early-stage renal tumors (T1a and T1b) at a tertiary hospital in India.

Methods This was a retrospective study of consecutive patients who underwent cryoablation for renal cell carcinoma (RCC) from November 2018 to June 2020. Cryoablation was performed under combined ultrasound and computed tomography guidance using a helium-argon cryoablation system. Tumor number, size, location, nephrometry score, relationship of the tumor with pelvicalyceal system, and adjacent organs were tabulated, and technical and clinical success evaluated. Early and late recurrence and complications were also assessed.

Results Eleven patients (median age: 62 years) with 11 tumors underwent cryoablation. The mean tumor size was $2.58 \mathrm{~cm}$ (range: $1.62-5.62 \mathrm{~cm}$ ) with 10 lesions being T1a and one lesion T1b. Tissue sampling was done in 9/11 patients, 3 were papillary RCC and the rest, clear cell RCC. In two patients, the tumor was completely endophytic, three patients had partially endophytic tumors while 6 patients had exophytic lesions. The median nephrometry score was 6 (range: $4-11$, Mode 4). Technical success was achieved in all patients. Complete response was achieved in $81 \%(9 / 11)$ of the patients at 1-month follow-up. Median follow-up period was 6 months. Two patients showed residual disease on follow-up imaging at 1 and 3 months, respectively.

Conclusion Cryoablation is a promising, relatively new minimally invasive therapy for treating small renal tumors in India. It is safe, technically feasible, and shows excellent short-term efficacy.
\end{abstract}

article published online January 11, 2022
DOI https://doi.org/

$10.1055 / \mathrm{s}-0041-1740570$. ISSN 2457-0214.
(C) 2022. Indian Society of Vascular and Interventional Radiology. All rights reserved.

This is an open access article published by Thieme under the terms of the Creative Commons Attribution-NonDerivative-NonCommercial-License, permitting copying and reproduction so long as the original work is given appropriate credit. Contents may not be used for commercial purposes, or adapted, remixed, transformed or built upon. (https://creativecommons.org/ licenses/by-nc-nd/4.0/)

Thieme Medical and Scientific Publishers Pvt. Ltd., A-12, 2nd Floor, Sector 2, Noida-201301 UP, India 


\section{Introduction}

The annual incidence of renal cancer in India is around $1 \%{ }^{1}$ With advances in abdominal imaging, as well as its increased use for diagnosing other abdominal conditions, the number of incidentally detected renal cancers has increased. ${ }^{2}$ Partial nephrectomy is still the treatment of choice for early-stage renal cancers; however, local ablative therapies are gaining popularity as a means of conservative treatment for selected stage T1a and T1b tumors. ${ }^{3,4}$ Elderly patients not fit for surgery, or patients with compromised renal function, or those with only a solitary kidney are candidates for nephron sparing local ablative techniques. ${ }^{5}$ Local ablative techniques include chemical, thermal, and nonthermal methods. Thermal techniques are further classified into heat-based (radiofrequency ablation [RFA], microwave ablation, highfrequency ultrasound [US], laser ablation) and cold-based (cryoablation) techniques. ${ }^{6,7}$

During cryoablation the targeted diseased tissue, with a surrounding margin of healthy parenchyma, is rapidly frozen in situ. This devitalized tissue is then allowed to slough over time, with healing by secondary granulation. Cryoablation has the lowest incomplete ablation rate (4.8\%), lower complication rate $(1.8 \%)$, and it promotes faster recovery and discharge from hospital among all other ablative techniques for renal tumors. ${ }^{8-10}$ Furthermore, a renal biopsy can be obtained in the same sitting prior to cryoablation. ${ }^{9}$

Although partial nephrectomy provides better oncological outcomes in terms of overall survival, local recurrence, and metastatic disease for renal tumors, cryoablation has been associated with a lower complication rate, ease of procedure, and better renal function preservation. ${ }^{7}$ Because of this, cryoablation is preferred whenever it is imperative to preserve renal function, such as in solitary kidney, multiple synchronous renal cell carcinoma (RCC), von Hippel-Lindau disease, familial RCC, or those with limited renal function. ${ }^{7,11}$ While popular in the western countries, the use of cryoablation for renal tumors has not been reported in India. The purpose of this study was to report short-term outcomes of cryoablation of early-stage renal tumors (T1a and T1b) at a tertiary hospital in India.

\section{Materials and Methods}

This is a retrospective study of consecutive patients who underwent percutaneous cryoablation of renal tumors from November 2018 to June 2020 at our institution. The study was approved by our ethics committee and informed written consent was obtained for the ablation. All patients received formal consultations by urology prior to the procedure. Patients having early-stage renal tumor (defined as T1a and T1b) were included. Patients with a renal tumor of T2 or more, and those with distant metastasis were excluded. The diagnosis of RCC was based on multiphasic contrastenhanced computed tomography (CT) or magnetic resonance imaging. Tumor number, size, location, relationship of the tumor with the pelvicalyceal system, and the adjacent organs were noted and ablative procedure was planned accordingly. Nephrometry scores based on the R.E.N.A.L. criteria (radius, exophytic/endophytic, nearness to collecting system or sinus, anterior/posterior, location relative to polar lines) were recorded for all masses.

\section{Percutaneous Cryoablation Procedure}

Cryoablation was performed using US and CT guidance or only CT guidance. The commercially available cryoablation system, Endocare Corporation cryosurgery system (Irvine, California, United States) was used. Patient positioning was based on the tumor location on preprocedure CT. Tumors located in the anterior renal cortex were treated by a lateral approach keeping the patient in lateral oblique position. Posteriorly located tumors were approached keeping the patient in prone position. Additional techniques such as hydrodissection were utilized for tumors located close to the critical structures such as the bowel and diaphragm. A distance of $<10 \mathrm{~mm}$ between the tumor and the critical structure was considered when deciding on the need for hydrodissection. This technique involved placing a $20 \mathrm{G}$ spinal/Chiba needle through a percutaneous approach under US/CT guidance along the outer margin of the renal mass and instilling sterile fluid (most commonly normal saline, or $5 \%$ dextrose) till satisfactory distance was achieved as documented by CT. The cryoablation procedure was performed subsequently.

The number of probes to be used was estimated based on the tumor size and geometry. A variable probe (V-probe cryoprobe, Endocare Corporation) with the shaft diameter of $2.4 \mathrm{~mm}$ and length of $15 \mathrm{~cm}$ was used in five cases. This probe allows creation of five different isotherms ranging in size from 1.5 to $5 \mathrm{~cm}$ by adjusting the slider. The length of the isotherm in an individual patient was decided based on the size of the lesion. In 6 patients a dedicated renal cryoprobe with shaft diameter of $1.7 \mathrm{~mm}$ and length $15 \mathrm{~cm}$ was used. These probes have fixed ice ball sizes with diameters of 32 , 18 , and $13 \mathrm{~mm}$ corresponding to isotherms of $0^{\circ} \mathrm{C},-20^{\circ} \mathrm{C}$, and $-40^{\circ} \mathrm{C}$. Two freeze-thaw cycles were performed with an initial freezing for 10 minutes, followed by thawing for 10 minutes and refreezing for 10 minutes and then passive thawing as per the recommended protocol. The ablation margin was extended $0.5 \mathrm{~cm}$ beyond the outermost boundary of the tumor. The ablation was considered adequate when the ice-ball covered the entire tumor along with $0.5 \mathrm{~cm}$ margin of the adjacent renal tissue.

\section{Follow-Up}

Biphasic contrast-enhanced CT/dynamic contrast-enhanced magnetic resonance or contrast-enhanced US were performed 1 month, 3 months, 6 months, and 1 year after ablation. The response evaluation was done using modified response evaluation criteria in solid tumors (mRECIST). In patients with residual or recurrent disease, a second line of treatment was planned after discussion with the referring physician.

\section{Outcome Measurement}

Technical success was defined as the ability to complete the ablative procedure as planned. Complete ablation was 
defined as the lack of any residual tumor on follow-up imaging at 1 month. Residual tumor was defined as asymmetric enhancing soft tissue at 3 months' follow-up imaging. Late local recurrence was defined as any enhancing soft tissue appreciated at subsequent imaging performed at 6 months or thereafter.

\section{Results}

Eleven patients with 11 RCCs underwent cryoablation during the study period. There were 8 men and 3 women. The median age was 62 years (range: $44-82$ years, mean: 62.4 years). The demographic characteristics of the patients are enlisted in - Table 1. All the patients had a single lesion in the kidney. Tissue sampling was obtained in 9 patients at the time of cryoablation. Three were papillary RCC and the rest, clear cell RCC. Two patients did not undergo biopsy due to high risk of bleeding. One patient had deranged coagulation profile due to chronic liver disease and the other patient had a very hypervascular tumor on imaging (suspected to be clear cell RCC). Ten patients (90.9\%) were stage 1 a (mean size: $2.58 \mathrm{~cm}$, range: $1.62-3.14 \mathrm{~cm}$ ) and one patient belonged to stage $1 \mathrm{~b}$ (size $5.62 \mathrm{~cm}$ ). The median nephrometry score was 6 (range: 4-11, Mode 4). Four patients (36.4\%) had their tumor close to the pelvicalyceal system (within $4 \mathrm{~mm}$ ). In two patients, the tumor was completely endophytic, three patients had partially endophytic tumors while 6 patients had exophytic lesions.

The most common indication for undergoing cryoablation was presence of medical comorbidities and old age which rendered them surgically unfit. Two patients, however, chose cryoablation over surgery as their preferred treatment modality. Deranged coagulation parameters in one patient precluded surgery. One patient had RCC in transplanted kidney while one patient had RCC in a solitary kidney following contralateral nephrectomy. Eight patients underwent cryoablation under local anesthesia, while three patients received conscious sedation using intravenous fentanyl and midazolam. Hydrodissection was performed in three patients during the procedure as the tumor was abutting adjacent bowel loops. Pyeloperfusion was not performed in any of our patients. In five patients, a single variable cryoprobe was used. Ablation was performed using two probes in five patients and three probes in one patient.

\section{Outcomes}

Technical success was seen in all patients. Median follow-up period in our study was 6 months (range: 3-12 months; mean: 6.2 months). Complete response based on mRECIST was achieved in $81 \%(9 / 11)$ of the patients at 1 -month follow-up. Two patients showed residual disease on follow-up imaging at 1 and 3 months, respectively, both had tumors within $4 \mathrm{~mm}$ from the collecting system. One of these patients was retreated with second session cryoablation. Follow-up imaging at 3 months after the second session revealed no residual tumor in the ablated bed. However, at 6 months' follow-up, a new enhancing mass appeared at a new site in the same kidney. This patient had

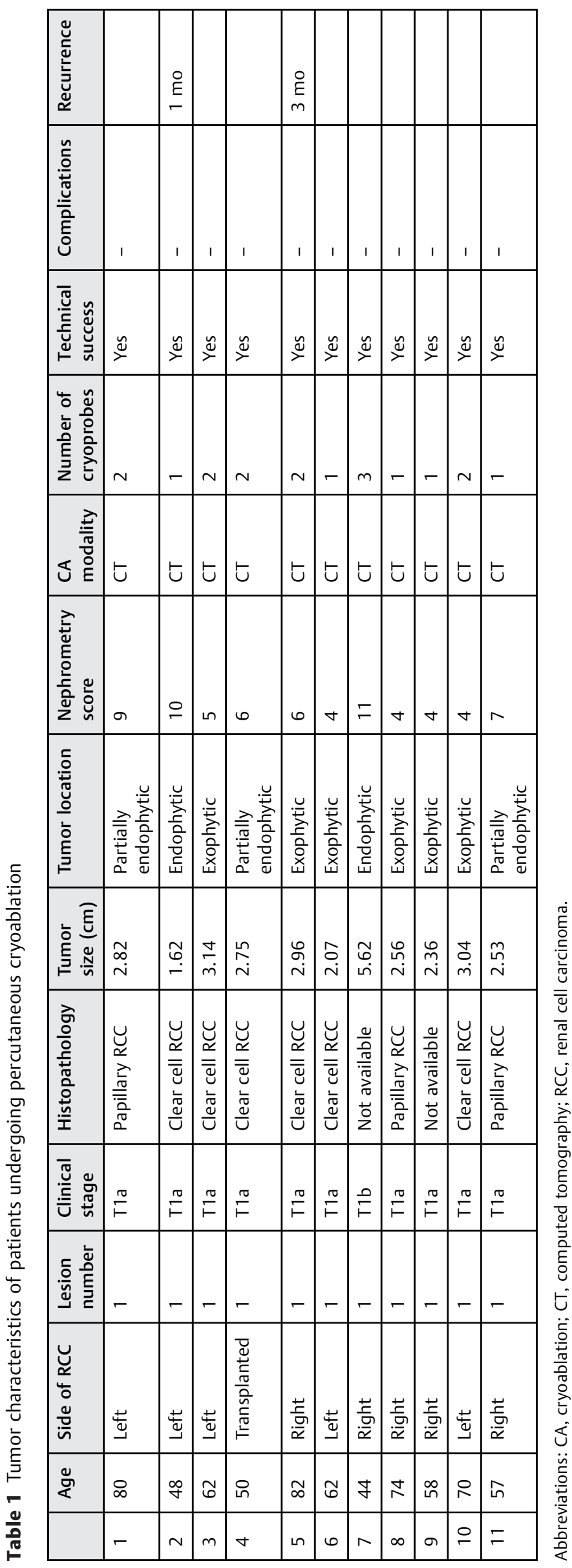


undergone radical nephrectomy for contralateral RCC 10 years ago and partial nephrectomy in the ipsilateral kidney prior to undergoing two sessions of cryoablation. Histopathology of both lesions revealed clear cell RCC. The other patient who showed recurrence was lost to follow-up. No local tumor progression or death was observed during the follow-up period in other patients. -Figs. 1-3 show representative cases.

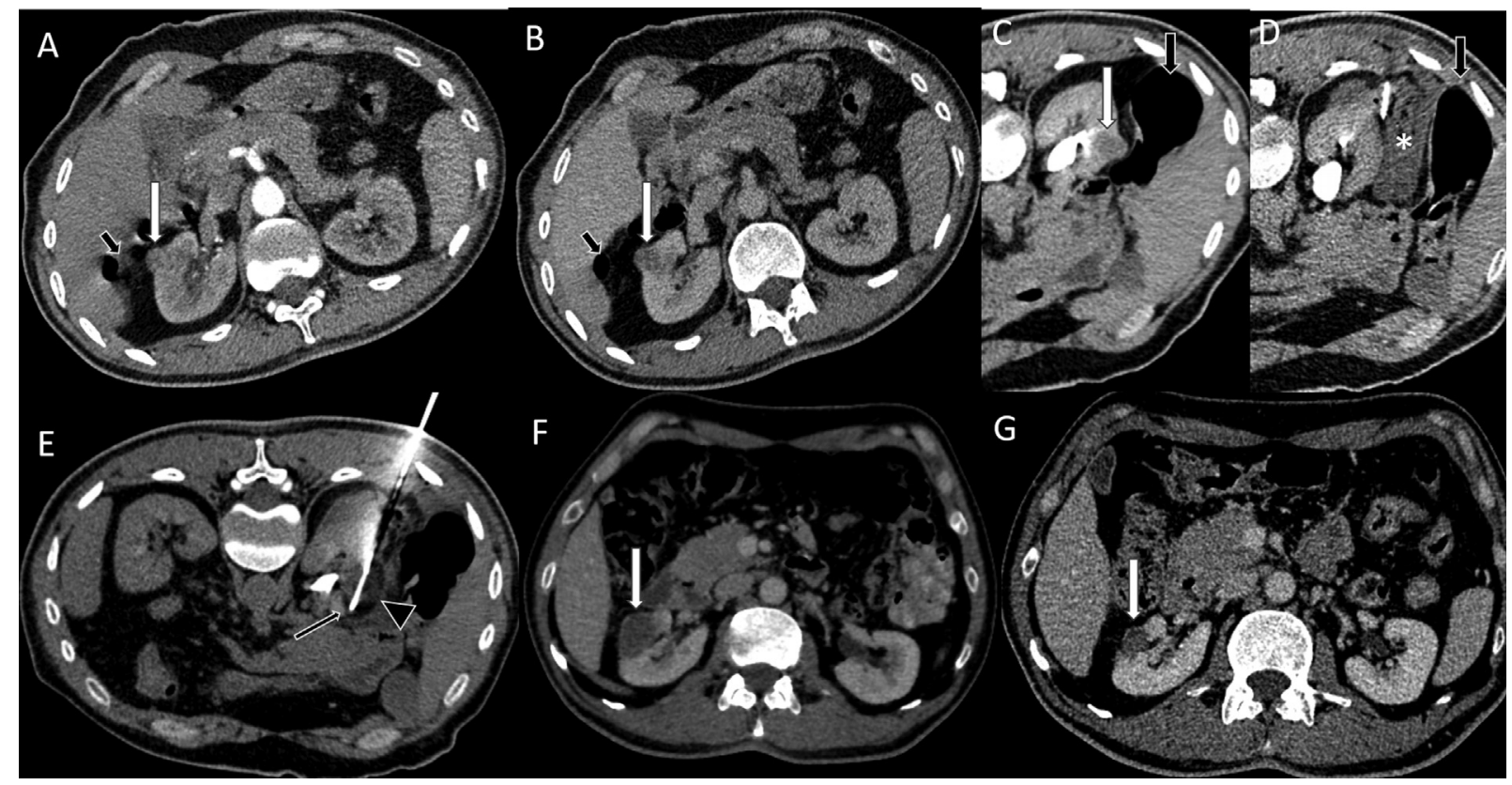

Fig. 1 A 57-year-old male with incidentally detected right renal mass. (A, B) Axial biphasic contrast-enhanced computed tomography (CECT) images show a $2.5-\mathrm{cm}$ well-defined partially endophytic solid renal mass in the anterior renal cortex of the right kidney which is hypoenhancing on arterial phase (A) as well as venous phase (B). The hepatic flexure was in close proximity to the renal mass (black arrow). (C) The patient was positioned prone for cryoablation. The renal mass (white arrow) was is in close relation to the ascending colon (black arrow). (D) Hydrodissection - 20G spinal needle was introduced under CT guidance and normal saline injected (white asterisk) displacing the ascending colon laterally (black arrow). (E) Cryoprobe was introduced under CT guidance with needle in situ (black arrow) and the lesion was ablated, forming a hypodense ice ball (black arrowhead). (F) Follow-up axial CECT at 1 month shows no enhancing lesion in the postop bed (white arrow). (G) Follow-up axial CECT at 6 months postablation - the ablated tumor has shrunken in size with no enhancing lesion (white arrow).

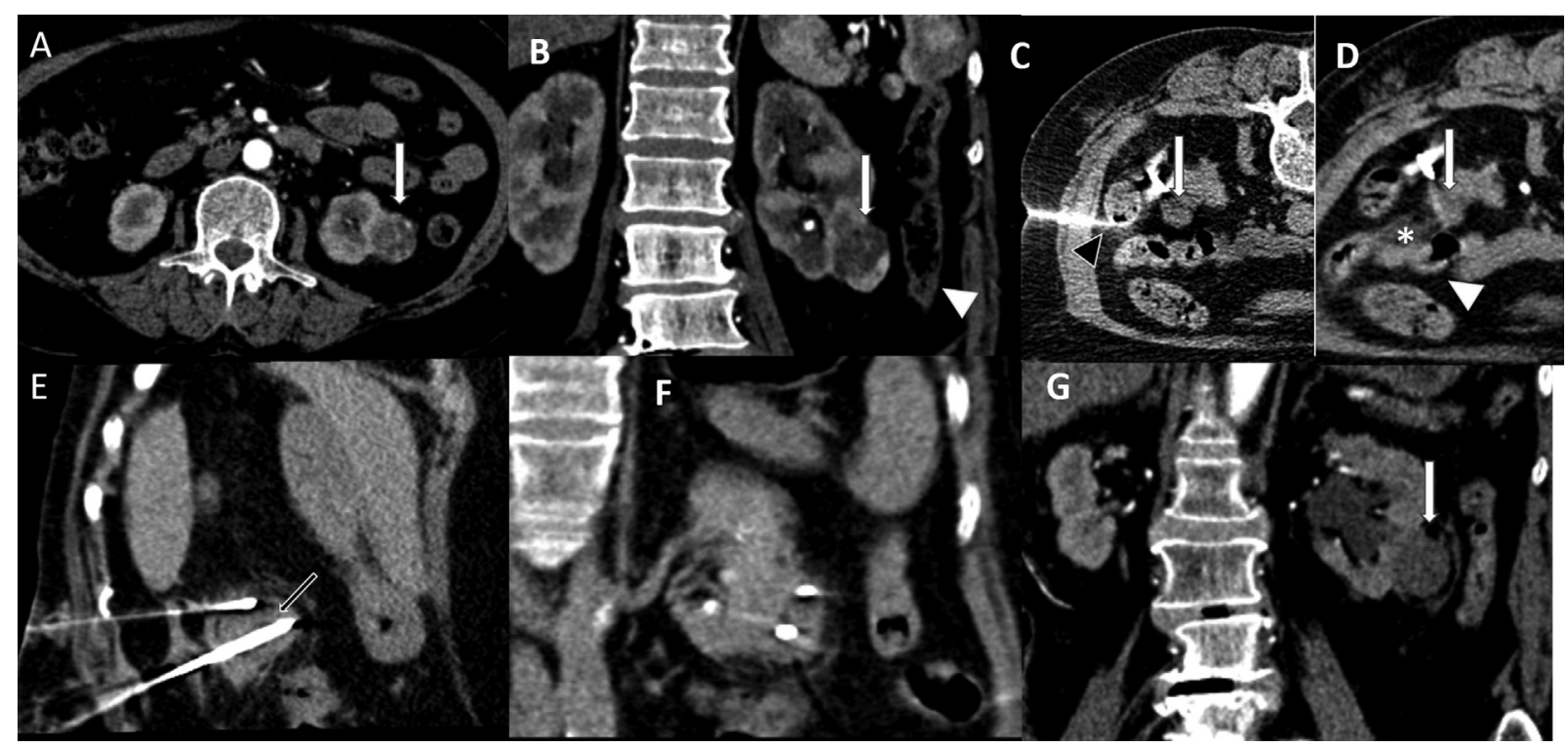

Fig. 2 A 70 years old female with incidentally detected left renal mass. (A, B) Axial biphasic contrast-enhanced computed tomography (CECT) shows a 3-cm exophytic heterogeneously enhancing mass lesion arising from the lower pole of the left kidney (white arrow) distant from the pelvicalyceal system; however, in close proximity with the descending colon (white arrowhead). (C, D) Patient was positioned prone for cryoablation. A 20G spinal needle (black arrowhead) was used to instill normal saline (asterisk in D) to create hydrodissection between the tumor (white arrow) and the descending colon (white arrowhead). (E, F) Sagittal and coronal oblique sections showing two cryoprobes (black arrow) introduced from posterior approach and the lesion cryoablated using 10 minutes cycles of freeze-thaw-freeze. (G) Follow-up axial CECT at 1 month shows no enhancing lesion in the postop bed to suggest residual disease (arrow). 


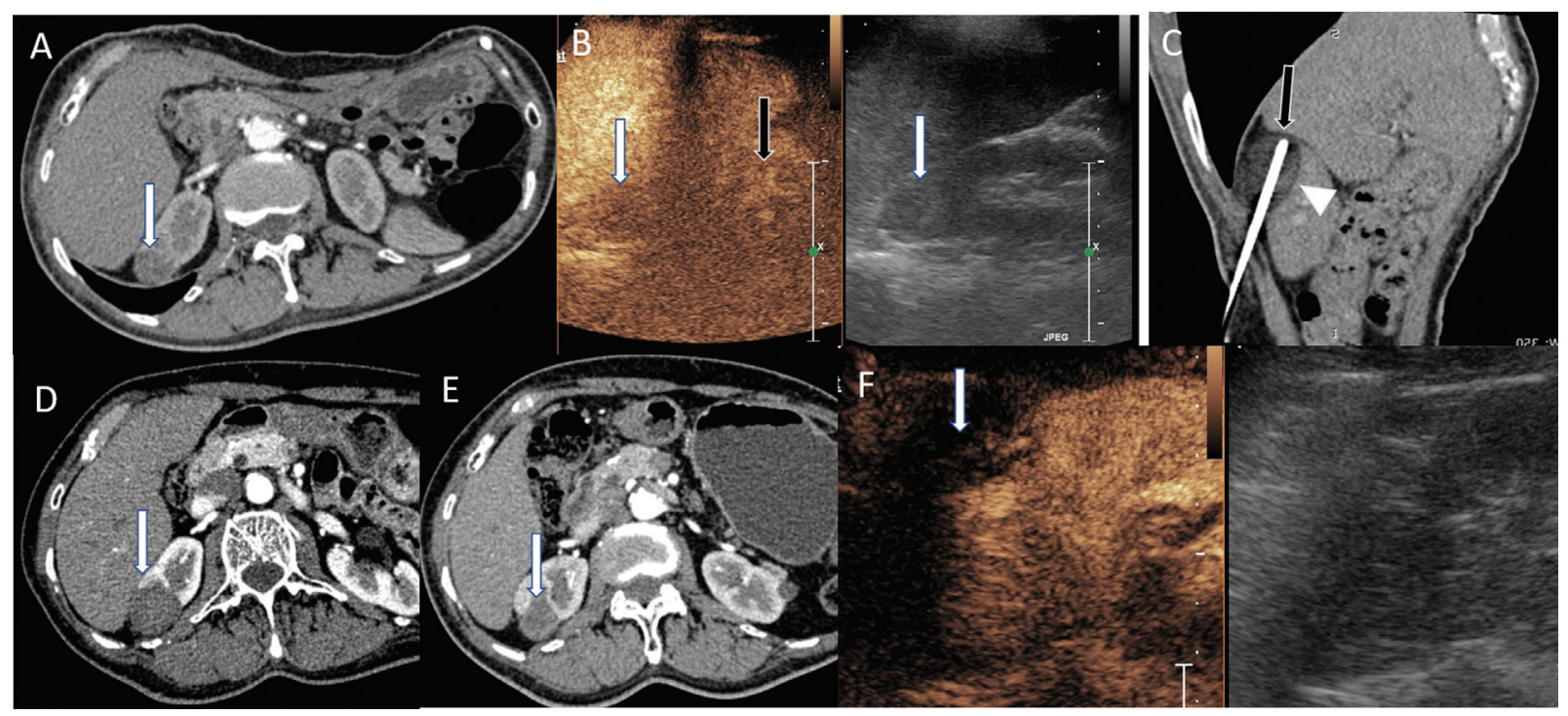

Fig. 3 A 74 years old male presented with right renal mass. (A) Axial contrast-enhanced computed tomography (CECT) image shows a hypoenhancing lesion arising from the upper pole of the right kidney. (B) Contrast-enhanced ultrasonography shows that the lesion is hypoenhancing (white arrow in B) to the normal renal parenchyma (black arrow in B). (C) The patient was positioned left laterally for the procedure, the cryoprobe (black arrow) was inserted under CT guidance and the lesion ablated using 10 minutes cycle of freeze-thaw-freeze. (D) Follow-up CECT at 1 month shows no enhancing lesion in the postop bed to suggest residual lesion. (E) Follow-up CT at 3 months shows that the ablated zone has reduced in size with no residual enhancing lesion. (F) Contrast-enhanced ultrasonography at 1 year postablation shows no vascularity in the ablated tumor bed.

\section{Complications}

No complications were encountered during the cryoablation procedure or during the hospital stay. No delayed complications were seen on follow-up.

\section{Discussion}

The two most commonly used ablative treatments for small renal masses (SRMs) include RFA and cryoablation. ${ }^{11}$ The major limitation of heat-based thermal ablation, especially RFA, is heat sink effect and small ablative zone. Heat sink effect prevents delivery of the radiofrequency energy to the tumor located adjacent to a major vessel. There is additional risk of thermal injury to the adjacent structures such as the renal pelvis, ureter, bowel, and diaphragm. Although techniques exist to address these problems, these add to the complexity of the procedure..$^{12,13}$

Cryoablation utilizes the Joule-Thomson effect to create lethal temperatures at the probe tips, ranging from $-20^{\circ} \mathrm{C}$ to $-40^{\circ} \mathrm{C}$ resulting in coagulative necrosis. Two synergistic mechanisms lead to cell death at this temperature, the intracellular (slow freeze) and the extracellular (fast freeze) mechanism which cause formation of ice crystals and are directly cytotoxic, leading to cell dehydration and rupture. ${ }^{9}$ This temperature is achieved by passage of high pressure argon gas through the cryoprobes. The volume of lethal tissue generated depends on the duration of freezing, size and number of probes used, and number of freeze-thaw cycles. Thawing can be active using helium gas or it can be passive. ${ }^{14}$ When the frozen tissue is thawed, it leads to microvascular occlusion resulting in indirect ischemia and cellular injury. ${ }^{9}$ Woolley et $\mathrm{a}^{15}$ showed similar results with active and passive thawing. In our study we used passive thawing.

The ideal candidate for percutaneous cryoablation is tumor less than $3 \mathrm{~cm}$ in size, posterior in location, partially exophytic, and with increased perinephric fat when the ablation is performed in elderly patients with comorbidities who cannot tolerate partial nephrectomy. ${ }^{5,9}$ Lethal temperature of normal kidney cells is $-20^{\circ} \mathrm{C}$ and for malignant cells is $-40^{\circ} \mathrm{C}$. The cryoprobe itself reaches temperatures of up to $-190^{\circ} \mathrm{C}$ and the edge of the ice ball has a temperate of $0^{\circ} \mathrm{C}$. Thus, the size of the ice ball is larger than the ablative zone. The $-20^{\circ} \mathrm{C}$ isotherm is $0.5 \mathrm{~cm}$ from the ice ball edge and $-40^{\circ} \mathrm{C}$ isotherm is $1 \mathrm{~cm}$ from the edge, thus a tumor ice ball margin of 0.5 to $1 \mathrm{~cm}$ should be the aim for adequate ablation. ${ }^{5,9}$

The main advantage of cryoablation over RFA is real-time visualization of the ice ball. Sonography allows monitoring of the anterior ablative margin of the ice ball, but the posterior edge of the ablation zone is obscured by the acoustic shadowing. On CT, the ice ball appears as a hypodense area and the entire extent of the ice ball can be appreciated. ${ }^{9}$ Additional advantages include lack of a heat sink effect as well as simultaneous application of multiple probes, creating a synergistic formation of ice ball that is larger than the simple additive effect of each cryoprobe. ${ }^{16}$ However, a similar "freeze-sink" effect is associated with cryoablation, whereby the thermal energy is carried away by blood vessels adjacent to endophytic and central tumors. Therefore, it is recommended to place tips of multiple cryoprobes close together in a central lesion and farther apart in posterior and exophytic lesions. ${ }^{5}$ Cryoablation is associated with higher risk of bleeding complications as compared with RFA since the vessels are not cauterized. ${ }^{17}$ However, we 
did not encounter any bleeding complication in any of our patients. The 2017 American Urological Association guidelines mention that both cryoablation and RFA have similar oncologic efficacy. $^{18}$

Recent studies have demonstrated cryoablation as a safe and efficacious locoregional therapy for the treatment of "difficult-to-treat" renal lesions. Historically, percutaneous approach has been cited as a relative contraindication to treat anterior renal masses due to their proximity with the bowel. ${ }^{19}$ Bodily et al $^{20}$ showed hydrodissection as a safe and efficacious technique to displace critical structures prior to introduction of cryoprobes for ablation, especially for anterior and lower pole renal masses. We successfully performed hydrodissection in three of our patients. Ileal loops required displacement in two of our patients (including one where ileal loop was abutting the transplanted kidney in the right iliac fossa) and colon in one patient. No injury was encountered during needle placement or fluid instillation.

Central lesions pose a challenge during ablative treatments due to increased rates of treatment failure and risk of injury to the collecting system. ${ }^{21}$ In our study, 2 out of 11 patients experienced recurrence during the follow-up period, both of which had tumors located within $4 \mathrm{~mm}$ from the collecting system. As compared with RFA, cryoablation is safer for treatment of centrally placed lesions near the collecting system and ureter since there is less risk of ureteral strictures and urinary leaks or fistula. ${ }^{22,23}$ None of the four patients with tumors located within $4 \mathrm{~mm}$ of the collecting system showed any urinary complications during the followup period.

Percutaneous ablative techniques offer a huge advantage over surgical approaches in negating the need for general anesthesia. Most investigators advocate and practice the use of local anesthesia or conscious sedation to avoid the risks and complications of general anesthesia. ${ }^{9}$ In addition, Allaf et $\mathrm{al}^{24}$ showed that percutaneous cryoablation required less analgesia than RFA. We believe that cryoablation is a minimally invasive daycare procedure and hence minimal anesthesia should be administered if required.

Cryoablation is increasingly being used to treat tumors larger than $3 \mathrm{~cm}$ with favorable short-term efficacy. Atwell et $\mathrm{al}^{25}$ reviewed the safety, efficacy, and short-term outcomes of large solid renal tumors (mean size: $4.2 \mathrm{~cm} \pm 1.1$ $\mathrm{cm}$ ) and found technical success in 38 out of 40 lesions. Follow-up imaging was available for 26 of the 40 patients and showed no local recurrence or tumor progression. In another case series of 37 patients with T1b lesions, technical success was achieved in $88.2 \%$ of the patients. ${ }^{26}$ After a mean followup period of 26.4 months, local recurrence was noted in 8 patients (23.5\%). Our study included one T1b lesion with largest dimension of $5.6 \mathrm{~cm}$ which was ablated using three cryoprobes. Short-term follow-up at 3 months showed no local tumor recurrence. No intraoperative or postprocedure complications were encountered and the patient was safely discharged.

Cryoablation has higher chances of bleeding than RFA likely because of thermal coagulation of vessels in RFA leading to better hemostasis. A recent advancement in the cryoprobe was introduced wherein a heating element is incorporated in the distal shaft of the probe for thawing the ice ball and subsequent track ablation. This gives the advantage of the superior tumor ablation of cryoprobe as well as heat-based track ablation of RFA. ${ }^{27}$ However, a recent study comparing the bleeding complications in patients undergoing cryoablation with and without heat-based track ablation shows no significant difference in major, minor, and overall bleeding complications between the two. ${ }^{27}$

There are several limitations to our study. It is a singlearm, retrospective study and the sample size is small. Biopsy was not performed in all patients prior to the ablation procedure and a head-to-head comparison could not be made with nephron sparing surgery due to limited sample size.

In conclusion, cryoablation is a promising, relatively new minimally invasive modality for treating small renal tumors in India. It is safe, technically feasible, and shows excellent short-term efficacy. It allows treatment of SRMs with no or minimal need of anesthesia and can be performed on a daycare basis. However, documenting long-term oncological efficacy requires larger studies and randomized control trials to incorporate it as a standard ablative procedure in clinical practice.

\section{Financial Disclosure}

None.

\section{Conflict of Interest \\ None declared.}

\section{Acknowledgments}

Authors would like to acknowledge Dr. Anupam Lal, Professor, and Dr. Harish Bhujade, Assistant Professor, Department of Radiodiagnosis; Dr. Girdhar Singh Bora, Associate Professor, Dr. Aditya Prakash Sharma, Assistant Professor, and Dr. Kapil Chaudhary, Assistant Professor, Department of Urology, for their invaluable clinical inputs and support.

\section{References}

1 Joshi A, Anand A, Prabhash K, et al. Kidney cancer demographics and outcome data from 2013 at a tertiary cancer hospital in India. Indian J Cancer 2017;54(04):601-604

2 Silverman SG, Israel GM, Trinh Q-D. Incompletely characterized incidental renal masses: emerging data support conservative management. Radiology 2015;275(01):28-42

3 Aoun HD, Littrup PJ, Jaber M, et al. Percutaneous cryoablation of renal tumors: is it time for a new paradigm shift? J Vasc Interv Radiol 2017;28(10):1363-1370

4 Georgiades CS, Rodriguez R. Efficacy and safety of percutaneous cryoablation for stage $1 \mathrm{~A} / \mathrm{B}$ renal cell carcinoma: results of a prospective, single-arm, 5-year study. Cardiovasc Intervent Radiol 2014;37(06):1494-1499

5 Seager M, Kumar S, Lim E, Munneke G, Bandula S, Walkden M. Renal cryoablation - a practical guide for interventional radiologists. BJR 2020

6 Fernando A, Fowler S, O'Brien TBritish Association of Urological Surgeons (BAUS) Nephron-sparing surgery across a nation - outcomes from the British Association of Urological Surgeons 2012 
national partial nephrectomy audit. BJU Int 2016;117(06): 874-882

7 Deng W, Chen L, Wang Y, et al. Cryoablation versus partial nephrectomy for clinical stage $\mathrm{T} 1$ renal masses: a systematic review and meta-analysis. J Cancer 2019;10(05):1226-1236

8 Campbell SC, Novick AC, Belldegrun A, et al; Practice Guidelines Committee of the American Urological Association. Guideline for management of the clinical T1 renal mass. J Urol 2009;182(04): 1271-1279

9 Allen BC, Remer EM. Percutaneous cryoablation of renal tumors: patient selection, technique, and postprocedural imaging. Radiographics 2010;30(04):887-900

10 Sterrett SP, Nakada SY, Wingo MS, Williams SK, Leveillee RJ. Renal thermal ablative therapy. Urol Clin North Am 2008;35(03):397-414 , viii viii

11 Zondervan PJ, Buijs M, De Bruin DM, van Delden OM, Van Lienden KP. Available ablation energies to treat CT1 renal cell cancer: emerging technologies. World J Urol 2019;37(03):445-455

12 Johnson DB, Solomon SB, Su L-M, et al. Defining the complications of cryoablation and radio frequency ablation of small renal tumors: a multi-institutional review. J Urol 2004;172(03): 874-877

13 Gervais DA, Arellano RS, McGovern FJ, McDougal WS, Mueller PR. Radiofrequency ablation of renal cell carcinoma: part 2, lessons learned with ablation of 100 tumors. AJR Am J Roentgenol 2005; 185(01):72-80

14 Erinjeri JP, Clark TWI. Cryoablation: mechanism of action and devices. J Vasc Interv Radiol 2010;21(8, Suppl):S187-S191

15 Woolley ML, Schulsinger DA, Durand DB, Zeltser IS, Waltzer WC. Effect of freezing parameters (freeze cycle and thaw process) on tissue destruction following renal cryoablation. J Endourol 2002; 16(07):519-522

16 Young JL, McCormick DW, Kolla SB, et al. Are multiple cryoprobes additive or synergistic in renal cryotherapy? Urology 2012;79 (02):484.e1-484.e6
17 Atwell TD, Carter RE, Schmit GD, et al. Complications following 573 percutaneous renal radiofrequency and cryoablation procedures. J Vasc Interv Radiol 2012;23(01):48-54

18 Campbell S, Uzzo RG, Allaf ME, et al. Renal Mass and Localized Renal Cancer: AUA Guideline. J Urol 2017;198(03):520-529

19 Hinshaw JL, Shadid AM, Nakada SY, Hedican SP, Winter TC III, Lee FT Jr. Comparison of percutaneous and laparoscopic cryoablation for the treatment of solid renal masses. AJR Am J Roentgenol 2008;191(04):1159-1168

20 Bodily KD, Atwell TD, Mandrekar JN, et al. Hydrodisplacement in the percutaneous cryoablation of 50 renal tumors. AJR Am J Roentgenol 2010;194(03):779-783

21 Wright AD, Turk TM, Nagar MS, Phelan MW, Perry KT. Endophytic lesions: a predictor of failure in laparoscopic renal cryoablation. J Endourol 2007;21(12):1493-1496

22 Sung GT, Gill IS, Hsu THS, et al. Effect of intentional cryo-injury to the renal collecting system. J Urol 2003;170(2 Pt 1):619-622

23 Brashears JH III, Raj GV, Crisci A, et al. Renal cryoablation and radio frequency ablation: an evaluation of worst case scenarios in a porcine model. J Urol 2005;173(06):2160-2165

24 Allaf ME, Varkarakis IM, Bhayani SB, Inagaki T, Kavoussi LR, Solomon SB. Pain control requirements for percutaneous ablation of renal tumors: cryoablation versus radiofrequency ablationinitial observations. Radiology 2005;237(01):366-370

25 Atwell TD, Farrell MA, Callstrom MR, et al. Percutaneous cryoablation of large renal masses: technical feasibility and short-term outcome. AJR Am J Roentgenol 2007;188(05): $1195-1200$

26 Gunn AJ, Joe WB, Salei A, et al. Percutaneous cryoablation of stage T1b renal cell carcinoma: safety, technical results, and clinical outcomes. Cardiovasc Intervent Radiol 2019;42(07):970-978

27 Schmit CH, Callstrom MR, Boorjian SA, et al. A comparison of bleeding complications in patients undergoing percutaneous renal cryoablation using cryoprobes with and without heat-based track ablation. J Vasc Interv Radiol 2018;29(06):874-879 